\title{
Minireview
}

\section{Methodological approaches in application of synthetic lethality screening towards anticancer therapy}

\author{
D Canaani*, \\ 'Department of Biochemistry, Faculty of Life Sciences, Tel Aviv University, Ramat Aviv, Tel Aviv 69978, Israel
}

\begin{abstract}
A promising direction in the development of selective less toxic cancer drugs is the usage of synthetic lethality concept. The availability of large-scale synthetic low-molecular-weight chemical libraries has allowed HTS for compounds synergistic lethal with defined human cancer aberrations in activated oncogenes or tumour suppressor genes. The search for synthetic lethal chemicals in human/mouse tumour cells is greatly aided by a prior knowledge of relevant signalling and DNA repair pathways, allowing for educated guesses on the preferred potential therapeutic targets. The recent generation of human/rodents genome-wide siRNAs, and shRNA-expressing libraries, should further advance this more focused approach to cancer drug discovery.

British Journal of Cancer (2009) I 00, 1213-1218. doi: 10.1038/sj.bjc.6605000 www.bjcancer.com

Published online 24 March 2009

(c) 2009 Cancer Research UK
\end{abstract}

Keywords: chemical synthetic lethality; chemical genetic interactions; genetic synthetic lethality; oncogenes; tumour suppressor genes; RNAi

Advances in our understanding of the molecular basis of cancer initiation, progression and metastatic expansion have raised hopes for quick translation of this new data for improved cancer detection, classification, monitoring and especially treatment. However, as pointed out by Varmus (2006), despite the significant progress in several individual cancers and the recent annual $1 \%$ decline in cancer-caused mortality rate in the United States, after several decades of steady increases, there are still major obstacles and challenges. The complexity of the problem is also manifested by the low success rate (for 1991-2000) in oncology drugs entering the clinical phase $(5 \%)$, as compared with the $11 \%$ average rate of success for all therapeutic areas (Kola and Landis, 2004). Unfortunately, in terms of costs, the vast majority of drug attrition in all disease areas is at late stages of clinical drug development (phases II and III). In 2000, the major causes of attrition in the clinic had been lack of sufficient efficacy (30\%) and toxicity $(30 \%)$ pertaining to both off-target and on-target toxicity (Kola and Landis, 2004).

Two recent reviews have dealt with the formidable problem of identification of drugs with improved efficiency and cancer selectivity (Benson et al, 2006; Collins and Workman, 2006). The former review by a Novartis Corp. group defined four tracks of cancer targets: genetics, synergy, lineage and host. These four subtypes of cancer targets can be viewed as 'dependencies', which constitute potential weaknesses - Achilles heels that are unique to cancer cells and can thus be exploited for therapy. The synergy track constitutes synthetic lethal genetic interactions.

The purpose of this minireview is to examine the methodologies that dictate the experimental plans and progress, in application of the concept of synthetic lethality for the identification of lead

*Correspondence: Professor D Canaani; E-mail: canaani@post.tau.ac.il Received 18 November 2008; revised 19 February 2009; accepted 4 March 2009; published online 24 March 2009 compounds/drugs, and to a limited extent also of targets, for cancer therapy. Noteworthy is the extensive review by Kaelin (2005) on 'The concept of synthetic lethality in the context of anticancer therapy', which presents the issue.

\section{THE BASICS OF SYNTHETIC LETHALITY}

Synthetic lethality describes a cellular condition in which two (or more) non-allelic and non-essential mutations, which are not lethal on their own, become deadly when present within the same cell. The synthetic lethal mutations may constitute partial mutations present together in a single linear essential pathway (Figure 1A), reside in parallel pathways leading to the synthesis of a common essential gene product (Figure 1B) or constitute independent parallel survival pathways each serving as salvage pathway in the absence of the other (see Figure 1C). Intermediate situations in which two mutant genes may generate a 'synthetic sickness' condition might also exist. These occurrences may become lethal and lose viability when combined with one or more additional non-essential mutation/s.

Although tentatively, the two mutant synthetic lethal genes are presumed to represent loss-of-function mutations, a condition of synthetic lethality between an overexpressed 'gene of interest' and a mutant null gene should also be taken into account (see below) and has been initially described in yeast as a 'synthetic dosage lethality' phenotype (Kroll et al, 1996).

The first methodology for genetic synthetic lethality screen has been initially developed in yeast, Saccharomyces cerevisiae (Bender and Pringle, 1991). A wild-type copy of the 'gene of interest', on an unstable episomal plasmid (containing an origin of DNA replication but no centromere), is introduced into yeast cells that are null for expression of this gene. Random mutagenesis of the entire yeast genome within these cells may inactivate a gene that is synthetic lethal with deficiency in the gene of interest. Under these 
conditions, retention of the episomal plasmid, which is otherwise spontaneously lost, and expression of the gene of interest become essential for survival. Plasmid loss or retention is detected by changes in colony pigmentation. Identification of the mutagenised synthetic lethal gene is accomplished by transforming the particular colony cell population with a wild-type yeast genomic library, while selecting for a change in pigment colour reflecting a loss for the need of the episomal plasmid (i.e., loss of the synthetic lethal condition). The availability of numerous yeast knockout mutants, and the implied added advantage of the method in revealing interactions that do not necessarily require physical interaction between the two gene products, has quickly made this method one of the most powerful in yeast genetics.

Two different modifications of the basic concept have created genetic synthetic lethality screens that are less tedious, more informative and thus suitable for genome-wide analysis. In one, termed synthetic genetic array (SGA), double mutants are generated by mating the query mutant haploid strain to a panel of yeast knockout mutant strains; unviable double-mutant meiotic progeny identifies synthetic lethal relationships (Tong and Boone, 2005). The alternative method named dSLAM for diploid-based Synthetic Lethality Analyzed by Microarray (Pan et al, 2004) takes advantage of TAGs representing DNA bar codes, which uniquely mark each yeast deletion allele. These TAGs are flanked by shared priming sequences for PCR. The query mutation is introduced into a pool of heterozygote diploid knockout yeast strains by integrative transformation. Following sporulation, the haploid single (mock transfection)- and double-mutant populations are selected. The presence of every individual deletion mutant within any of the two pools is assayed by measuring the relative abundance of its corresponding TAG-containing PCR product in genomic DNA prepared from each one of the pools, and competitively hybridising to a TAG oligonucleotide microarray. The disappearance of a particular TAG from the double-mutant cell population then may point to a synthetic lethal interaction. The addition of the SGA and DSLAM versions of the yeast genetic synthetic lethality screening enables identification of gene networks and cellular pathways that 'buffer' each other biologically (Ooi et al, 2006).

The first to suggest the usage of synthetic lethality screening, chemical as well as of genetic, for the development of cancer therapy have been Hartwell and Friend (Hartwell et al, 1997). However, figuring that the state of genetic manipulations in mammalian and human-cell systems, in particular, was not ripe yet for genome-wide genetic synthetic lethality screening (a situation that has changed dramatically with the introduction of synthetic siRNAs in 2001), they suggested the use of model genetic systems, such as yeast, the nematode Caenorhabditis elegans and the Drosophila melanogaster fruit fly.

\section{CHEMICAL SYNTHETIC LETHALITY SCREENING IN YEAST}

In their search for cancer-specific genetic changes, which could form potential selective therapeutic targets, Hartwell and Friend relied on the fact that one of the hallmarks of cancer is genetic instability. This instability is primarily caused by defects in DNA repair, in cell cycle checkpoints and in other cell cycle controls. Moreover, because these processes have been well conserved between yeast and humans, these investigators used a panel of up to 70 isogenic strains, each defective in either DNA repair or cell cycle control gene/genes, for the systematic testing of the 33 most common FDA-approved anticancer cytotoxic drugs (Hartwell et al, 1997; Simon et al, 2000). This screen identified targets which sensitise chemotherapy drugs administered to yeast. The bifunctional alkylating agent cisplatin, for example, was killing 100-fold better $\left(\mathrm{IC}_{50}\right.$ decreased by two logs) in the presence of defects in
A Partial ablation of two enzymes located on one essential pathway

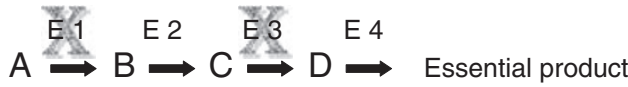

B Ablation of two enzymes located on parallel pathways leading to a common essential product

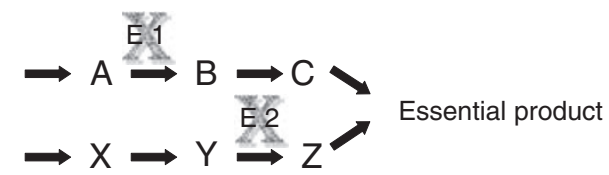

C Ablation of two enzymes on independent survival pathways leading to synthetic lethality

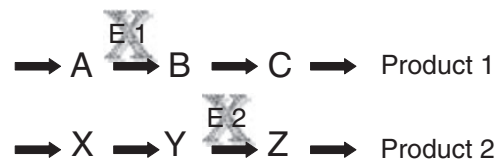

Figure I (A) Partial ablation of two enzymes located on one essentia pathway. (B) Ablation of two enzymes located on parallel pathways leading to a common essential product. (C) Ablation of two enzymes on independent survival pathways leading to synthetic lethality. Three modes of cell survival pathways amenable for analysis by the synthetic lethality screening approach.

either the Rad6 or Rad18 post-replication repair genes. Similarly, defects in the Rad50, Rad51 or Rad52 double-strand break repair through homologous recombination (HR) augmented by almost two logs the killing efficiency by topoisomerase inhibitors, such as camptothecin (Topo I) and mitoxantrone (Topo II). Obviously, these chemotherapeutic-sensitising links call to be tested with regard to their human orthologues in normal and tumour-derived cell lines.

Also screened were more than 85000 compounds from the collection of the Developmental Therapeutics Program (DTP) branch of NCI (Dunstan et al, 2002). This large-scale chemical screen (which was part of the Seattle project based at the Fred Hutchinson Cancer Research Center) identified 126 compounds that were selectively toxic to yeast cells defective in double-strand break repair ( $\operatorname{rad} 50 / 52$ specific). Out of these, 87 compounds were known or closely related to known topoisomerase inhibitors, and 39 were of no known function. The manuscript relating to this screen (Dunstan et al, 2002) described the analysis of eight such compounds. The rest of the information (updated to 2002) was made available at the DTP website http://www.dtp.nci. nih.gov/yacds/index.html under 'NCI Yeast Anticancer Drug Screen'. Among the eight compounds analysed, seven were toxic to mammalian cells; five classified as topoisomerase I poisons and two as topoisomerase II inhibitors. This feasibility/proofof-concept study showed, in part, the pros and cons in using a model organism for the identification of anticancer drugs in humans.

A boost to the usage of chemical synthetic lethality screens in yeast has been achieved by integrating drug-sensitivity profiles with those of the large-scale genetic interaction data obtained through genome-wide genetic synthetic lethality screens performed by either SGA (Tong and Boone, 2005) or dSLAM (Pan et al, 2004; Ooi et al, 2006). The clustering of the two profiles links compounds to their protein targets and/or target pathways (Parsons et al, 2004; Lopez et al, 2008).

Recently, the screening of chemical libraries in panels of knockout collections of $S$. cerevisiae, for unravelling chemical synthetic lethality relationships, has been complemented through usage of collections of mutant yeast strains generated by meiotic recombination (Perlstein et al, 2006). 
CHEMICAL SYNTHETIC LETHALITY IN MAMMALIAN CELLS

\section{Methodology: high-throughput chemical screening}

Over the past decade, three general methods of mammalian-cellbased HTS for synthetic lethal compounds have been reported. The first is the classical screening for genotype-specific inhibitors that are differentially toxic to mutant cell lines grown in multiwell plates. For example, the group led by Stuart Schreiber has screened marine sponge extracts for replication inhibitory activity specific for mouse embryo fibroblasts with either $\mathrm{p} 53^{-I-}$ or $\mathrm{p} 21^{\mathrm{kipl}-I-}$ genotype $v s$ wild type. The assay monitored BrdU incorporation by cytoblotting (Stockwell et al, 1999). This classical differential toxicity screening method is subject to several well-known disadvantages: errors due to differences in cell density relating to variations in cell-plating efficiency or growth rate; mistakes resulting from differences in microenvironment growth conditions among plates and wells (particularly regarding circumference effects); the inability of most cell viability assays for multiple readings from zero time on; and the high costs of tissue culture ware and time required for growth analysis. Two methods were independently developed to circumvent some of these shortcomings. In one, developed by Kinzler and co-workers (Torrance et al, 2001), two isogenic human colon cancer cell lines, each marked by a distinct mutant GFP gene, were co-cultured into the same wells in multiwell plates. The two isogenic cell lines differed in that in one the mutant $K$-Ras oncogene allele was deleted by HR to generate a null allele. The co-culturing of the two isogenic cell lines supplied a highly important internal control and created an 'even growth environment' that minimised potential differences in cell density. The tagging of each cell line by a distinct GFP mutant (capable of double-label reading) allowed for multiple time-point assessments of the relative cell viability through GFP fluorescence monitoring. Yet, this method still has two cell lines that may have different growth rates and the co-culturing of which might render one sensitive to growth-enhancing/inhibitory paracrine signals secreted by the other.

The second recently developed method for high-throughput chemical (and genetic) synthetic lethality screening was generated for human cells (Simons et al, 2001a) and for mouse embryo fibroblasts (Einav et al, 2003), by my research group. In trying to establish a direct method for finding bona fide synthetic lethality relationships between a chemical and a mutated gene (or between two mutated genes in the genetic screen), we resorted to the concept of the original equivalent yeast method, that is, complementation expression of a gene of interest through a lowcopy-number unstable episomal replication, in which retention of the episome is forced either by a selectable marker or by synthetic lethal pairing with another gene. Under such synthetic lethal conditions, retention of the episomal plasmid, expressing the wildtype gene of interest, becomes indispensable for viability. Tagging of the chromosomal host DNA and the episomal 'survival plasmid' with different GFP variants compatible for double-label fluorescence reading allows for the normalised quantitative detection of the episomal plasmid. Microtitre plates were seeded with either HPRT1-deficient human HT1080 fibrosarcoma cells or HPRT1 null mouse embryo fibroblasts as model systems. We have then shown using HPRT1-expressing Epstein-Barr Virus-based plasmid replicons that one can screen and detect synthetic lethal chemicals (Simons et al, 2001a; Einav et al, 2003) as well as genes (Simons et al, 2001b; Einav et al, 2005). This purine biosynthesis de novo and salvage pathways leading to the synthesis of the common essential product GMP are an example for the model shown in Figure 1B. The major advantage of this system is that it utilises a single cell line that is thus immune to variations in cell growth characteristics of isogenic cell line pairs or their putative paracrine interactions with each other within the same well. Also, the assay's direct proof for a synthetic lethality condition (i.e. retention of the 'survival plasmid') spares a lot of controls needed when using the two other methods that are based on the principle of differential toxicity. On the basis of our experience, it is worthy to note that usage of the GFP variant ratio tends to distort results somewhat. At the same time, other viability detection methods, such as respiration rates or ATP pools, may also harbour the disadvantage of non-linearity, particularly in response to increased cell density. It is for this reason that all HTS methodologies need to be well calibrated to ensure linear responsiveness towards all system parameters. Usage of unstable GFPs that have higher turnover rates may potentially improve matters. Yet, in our model system, the expression level of a GFP variant with a half-life time of $4 \mathrm{~h}$ was too low for accurate monitoring. Also, while attempting to use episomal systems established in either breast carcinoma-derived cell lines or MEFs, for screening chemical libraries containing very large numbers (thousands) of chemicals, we observed loss of episomes. This is to say that continuous large-scale cell propagation may lead to the loss of the episome, despite selection for a dominant selectable marker gene encoded by the episome. Concomitantly, there is an increased rate of integration into the host chromosome, supporting resistance to the selectable marker. In lieu of the above, the use of the episomal system should be restricted to validation experiments or small-scale experiments in which the episome containing transfectant cell line is limited to a low passage number following its generation.

\section{Screening for chemicals synthetic lethal with activated oncogenes}

Starting from an isogenic pair of mouse mammary epithelial cell lines, one of which is stably expressing an ectopic neu/Her2 oncogene, Leder and co-workers screened a chemical library containing 16000 compounds. One molecule, F16, a lowmolecular-weight delocalised lipophilic cation, preferentially accumulated in the inner mitochondrial matrix due to its elevated membrane potential $\left(\Delta \psi_{\mathrm{m}}\right)$ (Fantin et al, 2002). This accumulation led to the perturbation of mitochondrial homoeostasis and eventual preferential cell death of the neu-overexpressing cells. Further study has shown a linkage between F16 activity and transformed cells displaying increased mitochondrial membrane potential, rather than neu overexpression per se. In terms of its potential cancer therapy usage, F16 is still at the discovery stage (Fantin and Leder, 2006).

Another HTS of chemical libraries centred on tumour cells overexpressing the Ras oncogene. Using their human colon cancer isogenic cell lines in which a mutant $K$-Ras allele has been deleted by targeted HR in one of the two cell lines (see above), the groups led by Kinzler and Vogelstein have screened some 30000 lowmolecular-weight compounds (Torrance et al, 2001). Several compounds with up to six-fold greater toxicity towards the KRas-expressing cell line/s were identified. The cytidine nucleoside analogue was among the compounds showing activity in xenograft mouse models in vivo. Stockwell's group has performed two carefully designed large-scale differential toxicity screens for chemicals synthetic lethal with oncogenic Ras expressed in human cancer cells. A cell system, engineered by Weinberg's group, in which primary human forskin fibroblasts (BJ cells) were made immortal, transformed and tumourigenic by the constitutive expression of hTERT, SV40 large and small T oncogenes, and activated Ras ${ }^{\mathrm{V} 12}$ was used. Initially, 23550 compounds and later 47725 chemicals were screened for H-RAS/K-RAS-dependent synthetic lethality (Dolma et al, 2003; Yang and Stockwell, 2008, respectively). The first screen identified several known chemicals, as well as a novel compound named erastin, for 'eradicator of RAS and Small T-expressing cells', which displayed a modest eight-fold tumour selectivity in this in vitro model cell system, while causing a non-apoptotic cell death. 
The second screen (Yang and Stockwell, 2008) identified two compounds, named RSL5 and RSL3, for 'oncogenic-RAS-selective/ lethal compounds'. These cause, like erastin, a non-apoptotic, $M E K$-dependent and iron-dependent oxidative cell death. It is worth noting that cells transformed by oncogenic RAS have an increased iron content relative to their normal counterparts. Both RSL5 and erastin target VDAC3, a voltage-dependent anion channel 3. Interestingly, RSL3, whose mechanism of action is not clear yet, has been tested on the NCI60 panel of human tumour cell lines, and is found to have a unique sensitivity/resistance profile, as defined by the COMPARE algorithm, relative to the thousands of compounds already tested at NCI (Yang and Stockwell, 2008).

\section{Synthetic lethality with tumour suppressor gene deficiency}

The screening for chemicals synthetic lethal with a tumour suppressor gene deficiency, in isogenic pairs of tumour cell lines, poses a particular problem for the reference/control cell line. The generation of control 'tumour suppressor gene-corrected cell line', by restoring the normal function of an aberrant destabilised tumour suppressor protein with a stabilising ligand, is rarely possible (see the case for the p53 tumour suppressor by Boeckler et al, 2008). On the other hand, ectopic expression of the wild-type gene in the tumour suppressor-deficient cell line usually leads to cell cycle arrest and/or cell death (see below for the von HippelLindau (VHL) gene exception). Thus, researchers resorted to the usage of either reconstructed human model systems (such as BJ cells based) or intermediate-stage tumour cells, whose tumour suppressor gene/s product/s can be sequestered by siRNA/shRNA, or tumour suppressor-binding oncogenic proteins (SV40 large T antigen, HPV E6/E7, etc.). Alternatively, investigators have chosen to design their experimental screening systems based on understanding the consequences of particular tumour suppressor gene deficiency.

The case for VHL The VHL tumour suppressor gene is inactivated in about $75 \%$ of clear-cell renal cell carcinoma (CC-RCC) patients. Screening for chemicals or downregulated genes synthetic lethal with VHL inactivation was made possible by the observation by Kaelin's group that unlike most human tumour suppressor genes ectopic expression of VHL does not lead to inhibition of cell growth or of cell-cycle distribution in vitro, but rather to suppressing tumour formation in nude mice xenografts (Iliopoulos et al, 1995). One function of VHL is as an E3 ubiquitin ligase, which targets the hypoxia-inducible factor $\alpha$ (HIF- $\alpha$ ) transcription factor for oxygen-dependent proteolysis. Using in silico analysis of existing publicly available drug profiles of the NCI60 cell lines, chromomycin A3 (ChA3) was identified as being potentially lethal with VHL deficiency in CC-RCC. This HIF-dependent inhibition was first confirmed in vitro, followed by in vivo mouse xenograft validations (Sutphin et al, 2007). The same group (of Amato Giaccia) then screened a 64000 compound library against VHL-deficient $v s$ VHL-complemented RCC cells to identify the STF-62247 as a chemical synthetic lethal with VHL deficiency in vitro (25-fold difference in $\mathrm{IC}_{50}$ ), as well as in vivo. Unlike ChA3, STF-62247 cytotoxicity is HIF independent and engages autophagy induction (Turcotte et al, 2008). VHL deficiency and its synergistic lethal inhibitors represent examples of the model shown in Figure 1A.

The case for PTEN The PTEN (phosphatase and tensin homologue deleted on chromosome 10) tumour suppressor gene is the second most frequently mutated tumour suppressor gene after p53. PTEN antagonises the PI3K/AKT signal transduction pathway by dephosphorylating the $\mathrm{PIP}_{3}$ second messenger, which itself is formed from $\mathrm{PIP}_{2}$ by PI3K-dependent phosphorylation. In the absence of PTEN, AKT is activated by its recruitment to the cell membrane through its ligand - $\mathrm{PIP}_{3}$. The AKT kinase has many downstream substrates involved in apoptosis, cell proliferation and protein synthesis, one of which is mTOR (mammalian target of rapamycin). The synthetic lethality relationship between PTEN deficiency and mTOR-overexpression inhibition was discovered by showing an enhanced sensitivity of PTEN-deficient tumours to the mTOR inhibitor CCI779, a rapamycin homologue (Neshat et al, 2001; Podsypanina et al, 2001). Thus, the search for $\mathrm{PI} 3 \mathrm{~K} / \mathrm{AKT} / \mathrm{mTOR}$ inhibitors that are synthetic lethal, with PTEN deficiency in particular tumours, was intensified (see recent review by Yap et al, 2008). In a complementary elegant approach, the group led by Pamela Silver decided to try restoring at least some of the outcomes of PTEN function as a tumour suppressor gene by screening a renal carcinoma cell line (that is deficient in PTEN) for chemicals that relocalise the FOXOla transcription factor to the nucleus (Kau et al, 2003). AKT is a negative regulator of FOXOla. In PTEN null cells, AKT is activated and phosphorylates FOXOla, which is thus mislocalised to the cytoplasm, where it is unable to inhibit cell cycle progression. However, FOXOla is still capable of shuttling between the cytoplasm and the nucleus. Using a FLAG tagging for ectopic FOXOla expressed in the host PTEN-deficient tumour cells and an immunostaining-imaging assay, 18000 compounds were screened for imposing FOXOla nuclear retention. Forty-two lead compounds were identified, about half of which worked as general nuclear export inhibitors by targeting the CRM1 export transporter. Most of the other chemicals inhibited PI3K/AKT signalling. A large part of these 42 inhibitors blocked cell proliferation in a short-term assay (Kau et al, 2003).

The case for BRCA1/BRCA2 Our DNA is continually damaged by either endogenous activities, such as DNA replication and cellular free-radical generation, or exposure to environmental agents. This leads to diverse lesions, such as double- or single-strand breaks (DSBs and SSBs, respectively), base modifications and intrastrand or interstrand cross-links. Double-strand breaks are considered to be the most toxic of all DNA lesions. In mammalian cells, DSBs are generally repaired by non-homologous end-joining during $G_{0}, G_{1}$ and early $S$ phases of the cell cycle, and by HR during the $S-G_{2}$ phases of the cell cycle. The BRCA1 and BRCA2 human tumour suppressor genes are deficient in a subset of breast, ovarian and prostate cancers, and are normally required (among their other roles) for DSB repair by HR. The most common spontaneous DNA lesion in humans is base damage $(\sim 10000$ lesions per cell per day). Base excision repair (BER) is the major repair pathway for this lesion, with poly-(ADP) ribose polymerase 1 (PARP-1) enzyme being one of its essential repair ingredients. Inhibition of PARP-1 activity leads to persistence of SSBs (common intermediates in BER), which once encountered (in S phase) by a DNA-replication fork would result in fork stalling and potential DSBs. The formation of the latter can be repaired by HR. However, if impaired by BRCA1 or BRCA2 loss could result in cell cycle arrest and/or cell death. This potential synthetic lethal condition was tested and verified by the groups of Thomas Helleday and Alan Ashworth, while using PARP-1 inhibitors and BRCA1- or BRCA2-deficient cells (Bryant et al, 2005; Farmer et al, 2005). In view of recent discoveries of additional roles for PARP-1 in DNA repair, besides BER, the suggested mechanism for the synthetic lethality between PARP-1 inhibitors and BRCA1/BRCA2 deficiency, based on PARP-1-dependent ablation of BER, is but one of the several potential explanations (see Helleday et al, 2008; Lord et al, 2008). The homozygote BRCA1- or BRCA2-deficient cells were found to be 100 - to 1000 -fold more sensitive to PARP-1 inhibitors than the wild-type or heterozygote cell lines. The latter is particularly important, as normal, non-tumour cells in patients bearing BRCA1/BRCA2 mutations are heterozygotes. This monotherapy approach has a potentially large therapeutic window, with higher specificity and less side effects than standard cytotoxic chemotherapy. 
The proven synthetic lethality between deficiencies in the HR-dependent DNA repair genes, $B R C A 1$ or $B R C A 2$, and PARP1 inhibition has opened up several directions of clinical trials. Several PARP-1 inhibitors are currently in phase I or II of clinical monotherapy trials in $B R C A 1 / B R C A 2$-deficient breast or ovarian cancers (Helleday et al, 2008; Lord and Ashworth, 2008).

To augment the DNA-damage burden (on top of the spontaneous DNA damage), which cannot be repaired due to PARP-1 inhibition and deficiency in HR repair (BRCA1/BRCA2 deficiency), combination therapies are designed in which effective chemotherapeutics affecting DNA integrity are added together with PARP-1 inhibitors; this is a good example for the model shown in Figure $1 \mathrm{C}$, in which the outcome is DNA integrity affecting cell survival. Accordingly, early clinical trials are underway in which either one of the alkylating agents temozolomide, carboplatin, mitomycin or other DNA-damaging agents are added in combination with a PARP-1 inhibitor to patients with mutant BRCA1/ BRCA2 tumours (see details in Helleday et al, 2008 ; Lord and Ashworth, 2008). Also, in view of the fact that a fraction of the triple-negative $\left(\mathrm{ER} \alpha^{-}, \mathrm{PR}^{-}, \mathrm{HER} 2^{-}\right)$breast carcinomas display BRCA1/BRCA2 deficiency, phase-II clinical trials of this patient group, with PARP-1 inhibitors on their own or together with effective chemotherapeutics, are ongoing (http://clinicaltrials.gov).

\section{CONCLUSION}

The hopes for generating selective cancer drugs by virtue of their synthetic lethal interactions with tumour-specific aberrations are only beginning to materialise. It seems that early efforts in which model organisms, and yeast in particular, were used for drug discovery, have contributed primarily to methodologies, helping shape our experimental approaches in mammalian systems further on. Seemingly, the 'hit' rate in large-scale chemical synthetic lethality screening for anticancer compounds in mammalian systems turned out variable and low, requiring large resources of chemical libraries and automated screening facilities. The greater usage in the academia of HTS centres (such as those supported by the Molecular Libraries and Imaging Initiative and the Initiative for Chemical Genetics) and databases (such as PubChem and ChemBank) should certainly improve the prospects of finding new drug candidates.

\section{REFERENCES}

Bender A, Pringle JR (1991) Use of a screen for synthetic lethal and multicopy suppressee mutants to identify two new genes involved in morphogenesis in Saccharomyces cerevisiae. Mol Cell Biol 11: $1295-1305$

Benson JD, Chen YN, Cornell-Kennon SA, Dorsch M, Kim S, Leszczyniecka M, Sellers WR, Lengauer C (2006) Validating cancer drug targets. Nature 441: $451-456$

Boeckler FM, Joerger AC, Joggi G, Rutherford TJ, Veprintsev DB, Fersht AR (2008) Targeted rescue of a destabilized mutant of p53 by an in silico screening drug. Proc Natl Acad Sci USA 105: 10360-10365

Bryant HE, Schultz N, Thomas HD, Parker KM, Flower D, Lopez E, Kyle S, Meuth M, Curtin NJ, Helleday T (2005) Specific killing of BRCA2deficient tumours with inhibitors of poly(ADP-ribose) polymerase. Nature 434: 913 -917

Collins I, Workman P (2006) New approaches to molecular cancer therapeutics. Nat Chem Biol 2: 689-700

Dolma S, Lessnick SL, Hahn WC, Stockwell BR (2003) Identification of genotype-selective antitumor agents using synthetic lethal chemical screening in engineered human tumor cells. Cancer Cell 3: 285-296

Dunstan HM, Ludlow C, Goehle S, Cronk M, Szankasi P, Evans DR, Simon JA, Lamb JR (2002) Cell-based assays for identification of novel double-strand break-inducing agents. J Natl Cancer Inst 94: 88-94
The concept of searching for cancer drugs through synthetic lethality seems to be more rewarding when either the assay (FOXOla in PTEN ${ }^{-1-}$ ) or the potential target gene (mTOR in PTEN $^{-1-}$ ) can be decided upon based on data mining and prior knowledge of the normal and tumourigenic biological system. The most advanced case is the PARP-1 inhibitors, on their own or in combination with DNA-damaging chemotherapeutics, which are in phase-II clinical trials with breast/ovarian cancer patients having BRCA1/BRCA2 null tumours. Yet, it should be recalled that PARP1-inhibitory drugs were readily available since their usage in the nineties as chemosensitisers.

Although the identification of synthetic lethal lead compounds through large-scale chemical screening may be hard to come by because of chemical scarcity and large diversity, genetic synthetic lethality screening for gene target identification through siRNAs (Iorns et al, 2007) or constitutive/inducible shRNA libraries (Ngo et al, 2006; Schlabach et al, 2008, respectively) seems more promising. This is due, in part, to the limited number of human genes, and the increased efficiencies of the RNAi tools. The inclusion, on top of the above, of RNAi screens for sensitisers of cancer drugs (Whitehurst et $a l, 2007$ ), and initiatives such as the 'Connectivity Map', should further boost cancer targets and drug identification/validation.

At the same time, it may eventually turn out that the number of synthetic lethal interactions in human tumours/tumour cells is relatively limited, because of cell heterogeneity within tumours and multiplicity of compensatory survival pathways/subpathways, respectively. In this context, because resistance to therapy in many tumours may reside within cancer stem cells/tumourinitiating cells (CSCs/TICs), it will be extremely important to isolate CSCs and perform chemical and genetic synthetic lethality screens within this pure population so that CSC-specific drugs and targets could be identified.

\section{ACKNOWLEDGEMENTS}

This work was supported by grants awarded to DC by the Public Health Service National Cancer Institute and the German Israeli Foundation (GIF). I am grateful to Dr Iris Dotan for critical reading of this manuscript. I thank the anonymous reviewers for their helpful comments. I apologise to my colleagues, whose work was not cited due to space and reference limitations.
Einav Y, Agami R, Canaani D (2005) shRNA-mediated RNA interference as a tool for genetic synthetic lethality screening in mouse embryo fibroblasts. FEBS Lett 579: 199-202

Einav Y, Shistik E, Shenfeld M, Simons AH, Melton DW, Canaani D (2003) Replication and episomal maintenance of Epstein-Barr virus-based vectors in mouse embryonal fibroblasts enable synthetic lethality screens. Mol Cancer Ther 2: 1121-1128

Fantin VR, Berardi MJ, Scorrano L, Korsmeyer SJ, Leder P (2002) A novel mitochondriotoxic small molecule that selectively inhibits tumor cell growth. Cancer Cell 2: 29-42

Fantin VR, Leder P (2006) Mitochondriotoxic compounds for cancer therapy. Oncogene 25: 4787-4797

Farmer H, McCabe N, Lord CJ, Tutt AN, Johnson DA, Richardson TB, Santarosa M, Dillon KJ, Hickson I, Knights C, Martin NM, Jackson SP, Smith GC, Ashworth A (2005) Targeting the DNA repair defect in BRCA mutant cells as a therapeutic strategy. Nature 434: 917-921

Hartwell L, Szankasi P, Roberts C, Murray A, Friend S (1997) Integrating genetic approaches into the discovery of anticancer drugs. Science 278: $1064-1068$

Helleday T, Petermann E, Lundin C, Hodgson B, Sharma RA (2008) DNA repair pathways as targets for cancer therapy. Nat Rev Cancer 8: $193-204$ 
Iliopoulos O, Kibel A, Gray S, Kaelin WG (1995) Tumor suppression of the human von Hippel-Lindau gene product. Nat Med 1: 822-826

Iorns E, Lord CJ, Turner N, Ashwart A (2007) Utilizing RNA interference to enhance cancer drug discovery. Nat Rev Drug Discov 6: 556-568

Kaelin WG (2005) The concept of synthetic lethality in the context of anticancer therapy. Nat Rev Cancer 5: 689-698

Kau TR, Schroeder F, Ramaswamy S, Wojciechowski CL, Zhao JJ, Roberts TM, Clardy J, Sellers WR, Silver PA (2003) A chemical genetic screen identifies inhibitors of regulated nuclear export of a Forkhead transcription factor in PTEN-deficient tumor cells. Cancer Cell 4: 463-476

Kola I, Landis J (2004) Can the pharmaceutical industry reduce attrition rates? Nat Rev Drug Discov 3: 711-715

Kroll ES, Hyland KM, Hieter P, Li JJ (1996) Establishing genetic interactions by a synthetic dosage lethality phenotype. Genetics 143: $95-102$

Lopez A, Parsons AB, Nislow C, Giaever G, Boone C (2008) Chemicalgenetic approaches for exploring the mode of action of natural products. Prog Drug Res 237: 239-271

Lord CJ, Ashworth A (2008) Targeted therapy for cancer using PARP inhibitors. Curr Opin Pharmacol 8: 363 - 369

Lord CJ, McDonald S, Swift S, Turner NC, Ashworth A (2008) A highthroughput RNA interference screen for DNA repair determinants of PARP inhibitor sensitivity. DNA repair 7: 2010-2019

Neshat M, Mellinghoff IK, Tran C, Stiles B, Thomas G, Petersen R, Frost P, Gibbons JJ, Wu H, Sawyers CL (2001) Enhanced sensitivity of PTEN-deficient tumors to inhibition of FRAP/mTOR. Proc Natl Acad Sci USA 98: $10314-10319$

Ngo VN, Davis RE, Lamy L, Yu X, Zhao H, Lenz G, Lam LT, Dave S, Yang L, Powell J, Staudt LM (2006) A loss-of-function RNA interference screen for molecular targets in cancer. Nature 441: 106-110

Ooi SL, Pan X, Peyser BD, Ye P, Meluh PB, Yuan DS, Irizarry RA, Bader JS, Spencer FA, Boeke JD (2006) Global synthetic-lethality analysis and yeast functional profiling. Trends Genet 22: 56-63

Pan X, Yuan DS, Xiangg D, Wang X, Sookhai-Mahadeo S, Bader JS, Hieter P, Spencer F, Boeke JD (2004) A robust toolkit for functional profiling of the yeast genome. Mol Cell 16: 487-496

Parsons AB, Brost RL, Ding H, Li Z, Zhang C, Sheikh B, Brown GW, Kane PM, Hughes TR, Boone C (2004) Integration of chemical-genetic and genetic interaction data links bioactive compounds to cellular target pathways. Nat Biotechnol 22: $62-69$

Perlstein EO, Ruderfer DM, Ramachandran G, Haggarty SJ, Kruglyak L, Schreiber SL (2006) Revealing complex traits with small molecules and naturally recombinant yeast strains. Chem Biol 13: 319-327

Podsypanina K, Lee RT, Politis C, Hennessy I, Crane A, Puc J, Neshat M, Wang H, Yang L, Gibbons J, Frost P, Dreisbach V, Blenis J, Gaciong Z,
Fisher P, Sawyers C, Hedrick-Ellenson L, Parsons R (2001) An inhibitor of $\mathrm{mTOR}$ reduces neoplasia and normalizes p70/S6 kinase activity in Pten+/- mice. Proc Natl Acad Sci USA 98: 10320-10325

Schlabach MR, Luo J, Solimini NL, Hu G, Xu Q, Li MZ, Zhao Z, Smogorzewska A, Sowa ME, Ang XL, Westbrook TF, Liang AC, Chang K, Hackett JA, Harper JW, Hannon GJ, Elledge SJ (2008) Cancer proliferation gene discovery through functional genomics. Science 319: $620-624$

Simon JA, Szankasi P, Nguyen DK, Ludlow C, Dunstan HM, Roberts CJ, Jensen EL, Hartwell LH, Friend SH (2000) Differential toxicities of anticancer agents among DNA repair and checkpoint mutants of Saccharomyces cerevisiae. Cancer Res 60: 328-333

Simons AH, Dafni N, Dotan I, Oron Y, Canaani D (2001a) Establishment of a chemical synthetic lethality screen in cultured human cells. Genome Res 11: $266-273$

Simons AH, Dafni N, Dotan I, Oron Y, Canaani D (2001b) Genetic synthetic lethality screen at the single gene level in cultured human cells. Nucleic Acids Res 29: e100

Stockwell BR, Haggarty SJ, Schreiber SL (1999) High-throughput screening of small molecules in miniaturized mammalian cell-based assays involving post-translational modifications. Chem Biol 6: $71-83$

Sutphin PD, Chan DA, Li JM, Turcotte S, Krieg AJ, Giaccia AJ (2007) Targeting the loss of the von Hippel-Lindau tumor suppressor gene in renal cell carcinoma cells. Cancer Res 67: 5896-5905

Tong AH, Boone C (2005) Synthetic genetic array analysis in Saccharomyces cerevisiae. Methods Mol Biol 313: $171-192$

Torrance CJ, Agrawal V, Vogelstein B, Kinzler KW (2001) Use of isogenic human cancer cells for high-throughput screening and drug discovery. Nat Biotechnol 19: $940-945$

Turcotte S, Chan DA, Sutphin PD, Hay MP, Denny WA, Giaccia AJ (2008) A molecule targeting VHL-deficient renal cell carcinoma that induces autophagy. Cancer Cell 14: $90-102$

Varmus $\mathrm{H}$ (2006) The new era in cancer research. Science 312 $1162-1165$

Whitehurst AW, Bodemann BO, Cardenas J, Fergusen D, Girard L, Peyton M, Minna JD, Michnoff C, Hao W, Roth MG, Xie X-J, White MA (2007) Synthetic lethal screen identification of chemosensitizer loci in cancer cells. Nature 446: $815-819$

Yang WS, Stockwell BR (2008) Synthetic lethal screening identifies compounds activating iron-dependent, nonapoptotic cell death in oncogenic-RAS-harboring cancer cells. Chem Biol 15: 234-245

Yap TA, Garrett MD, Walton MI, Raynaud F, de Bono JS, Workman P (2008) Targeting the PI3K-AKT-mTOR pathway: progress, pitfalls, and promises. Curr Opin Pharmacol 8: 393-412 